\title{
DISCRETE HARDY'S TYPE INEQUALITIES AND STRUCTURE OF DISCRETE CLASS OF WEIGHTS SATISFY REVERSE HÖLDER'S INEQUALITY
}

\author{
S. H. SAKER* AND R. P. AGARWAL
}

Abstract. In this paper, we will prove a new discrete weighted Hardy's type inequality with different powers. Next, we will apply this inequality to prove that the forward and backward propagation properties (self-improving properties) for the general discrete class $\mathscr{B}^{p, q}$ of weights that satisfy the reverse Hölder inequality hold. As special cases, we will deduce the self-improving properties of discrete Gehring and Muckenhoupt weights. An example is considered for illustrations.

Mathematics subject classification (2010): 26D07, 40D25, 42C10 43A55,46A35, 46B15.

Keywords and phrases: Hardy's type inequality, discrete Gehring's class, discrete Muckenhoupt's class, higher summability, self-improving properties.

\section{REFERENCES}

[1] M. A. Arińo And B. Muckenhoupt, A characterization of the dual of the classical Lorentz sequence space $d(w, q)$, Proc. Amer. Math. Soc. 112 (1991), 87-89.

[2] G. Bennett And K.-G. Grosse-Erdmann, Weighted Hardy inequalities for decreasing sequences and functions, Math. Ann. 334 (2006), 489-531.

[3] J. Bober, E. CARneiro, K. Hughes And L. B. Pierce, On a discrete version of Tanaka's theorem for maximal functions, Proc. Amer. Math. Soc. 140 (2012), 1669-1680.

[4] A. BötTCHER AND M. SEYbold, Wackelsatz and Stechkin's inequality for discrete Muckenhoupt weights, Preprint no. 99-7, TU Chemnitz, (1999).

[5] A. BÖTTCHER AND M. SEYBOLD, Discrete one-dimensional zero-order pseudodifferential operators on spaces with Muckenhoupt weight, Algebra i Analiz 13 (2001), 116-129.

[6] L. D'Apuzzo And C. Sbordone, Reverse Hölder inequalities: a sharp result, Rend. Mat. Appl. (VII) (1990), 357-366.

[7] F. W. GEHRING, The $L^{p}$-integrability of the partial derivatives of a quasiconformal mapping, Bull. Amer. Math. Soc. 97 (1973), 465-466.

[8] F. W. GEHRING, The $L^{p}$-integrability of the partial derivatives of a quasi-conformal mapping, Acta Math. 130 (1973), 265-277.

[9] R. Hunt, B. Muckenhoupt, And Richard WheEden, Weighted norm inequalities for the conjugate function and Hilbert transform, Trans. Amer. Math. Soc. 176 (1973), 227-251.

[10] A. A. KorENOVS KII, The exact continuation of a reverse Hölder inequality and Muckenhoupt's conditions, Math. Notes 52 (1992), 1192-1201.

[11] F. LIU, Endpoint regularity of discrete multisublinear fractional maximal operators associated with $l^{1}$-balls, J. Ineq. Appl. 2018 (2018), 33.

[12] K. J. Hughes, Arithmetic analogues in harmonic analysis: Results related to Waring's problem, Thesis (Ph. D.), Princeton University, 2012, 112 p.

[13] Yu. I. LyubARS KiI AND K. SeiP, Complete interpolating sequences for Paley-Wiener spaces and Muckenhoupt's $\left(A_{p}\right)$ condition, Rev. Mat. Iberoamericana 13 (1997), 361-376.

[14] J. MADRID, Sharp inequalities for the variation of the discrete maximal function, Bull. Austr. Math. Soc. 95 (2017), 94-107. 
[15] A. Magyar and E. M. Stein And S. Wainger, Discrete analogues in harmonic analysis: Spherical averages, Ann. Math. 155 (2002) 189-208.

[16] N. A. Malaksiano, The exact inclusions of Gehring classes in Muckenhoupt classes, (Russian), Mat. Zametki 70, 5, 2001, 742-750; translation in Math. Notes, 70, 5-6, 2001, 673-681.

[17] B. MucheKnhoupt, Weighted norm inequalities for the Hardy maximal function, Trans. Amer. Math. Soc. 165 (1972), 207-226.

[18] B. S. PAVlov, Basicity of an exponential system and Muckenhoupt's condition, Dokl. Akad. Nauk SSSR 247:1, (1979), 37-40; English translation in Sov. Math. Dokl. 20:4, (1979), 655-659.

[19] A. Popoli, Optimal integrability in $B_{p}^{q}$ classes, Matematiche (Catania), 52-I (1997), 159-170.

[20] A. POPOLI, Sharp integrability exponents and constants for Muckenhoupt and Gehring weights as solutions to a unique equation, Ann. Acad. Sci. Fenn. Math. 43 (2018), 785-805.

[21] A. Popoli, Limits of the Ap-constants, J. Math. Anal. Appl. 478 (2), (2019), 1218-1229.

[22] S. H. SAKER AND I KUBIACZYK, Higher summability and discrete weighted Muckenhoupt and Gehring type inequalities, Proc. Ednb. Math. Soc. 62 (2019), 949-973.

[23] S. H. SAKER, S. S. RABIE, G. AlNemer, M. ZAKARYA, On structure of discrete Muckenhoupt and discrete Gehring classes, J. Ineq. Appl. 2020 (1), 1-18.

[24] S. H. SAKer, M. KRnić, The weighted discrete Gehring classes, Muckenhoupt classes and their basic properties, Proc. Amer. Math. Soc. 149 (2021), 231-243.

[25] S. H. Saker, D. O'Regan And R. P. Agarwal, Self-improving properties of discrete Muckenhoupt weights, Analysis (submitted).

[26] S. H. Saker, S. S. Rabie, J. Alzabut, D. O'Regan, R. P. Agarwal, Some basic properties and fundamental relations for discrete Muckenhoupt and Gehring classes, Adv. Diffe. Eqns. 2021 (1), $1-22$.

[27] S. H. Saker, S. S. Rabie, R. P. Agarwal, Properties of a generalized class of weights satisfying reverse Hölder's inequality, J. Fun. Spaces 2021, Volume 2021, ID 5515042, doi.org/10.1155/2021/5515042.

[28] C. Sbordone and G. ZecCa, The $L^{p}$-Solvability of the Dirichlet Problem for Planar Elliptic Equations, Sharp Results, J. Fourier Anal Appl. 15 (2009), 871-903.

[29] J.-O. Strömberg And A. Torchins Ky, Weighted Hardy Spaces, Lecture Notes in. Math. 1381, Springer, Berlin (1989). 